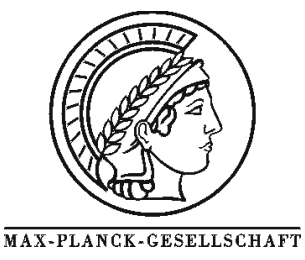

\title{
Electron beam induced changes in transition metal oxides
}

\author{
D. S. Su*
}

Department of Inorganic Chemistry, Fritz-Haber-Institute of the MPG, Faradayweg 4-6, 14195 Berlin, Germany

* Corresponding author: e-mail dangsheng@,fhi-berlin.mpg.de, phone +49 3084135406

Submitted 20 January 2002; accepted 14 May 2002

\begin{abstract}
Electron beam induced changes in maximum valence transition metal oxides $\mathrm{V}_{2} \mathrm{O}_{5}, \mathrm{MoO}_{2}$ and $\mathrm{TiO}$ (anatase) were studied by means of electron energy-loss spectroscopy and electron diffraction in a transmission electron microscope. For $\mathrm{V}_{2} \mathrm{O}_{5}$, the observed chemical shifts of the $L$-edge reveal the reduction of $\mathrm{V}^{5+}$ to $\mathrm{V}^{2+}$, while its structure changes from orthorhombic $\mathrm{V}_{2} \mathrm{O}_{5}$ to cubic $\mathrm{VO}$. The orthorhombic $\mathrm{MoO}_{3}$ can be reduced to a phase with an oxidation state lower than that in $\mathrm{MoO}_{2}$. This phase has a cubic or tetragonal structure with a $=\mathrm{c}=0.408 \mathrm{~nm}$. For TiO ${ }_{2}($ anatase), no noticeable changes in the intensity of the $\mathrm{O} K$-edge can be observed. The main structure symmetry prevails during the electron irradiation.
\end{abstract}

Keywords: Transition metal oxides, Reduction, Oxidation state, Electron beam, Transmission electron microscopy

\section{Introduction}

High-voltage transmission electron microscopes $(100 \mathrm{kV}-$ $400 \mathrm{kV}$ ), equipped with electron energy-loss spectrosmeter (EELS), are widely used in structural and electronic analysis of solid state materials. Fast electrons interact with atomic nucleus (elastic) and atomic electrons (inelastic) that provide image contrast and structural information in electron microscopy. However, such interactions can also initiate destructive processes through which the structure/chemistry of the sample under investigation is changed. These electron beam induced structural changes, usually called radiation damage, cause artefacts in the electron micrograph or in the recorded EEL-spectrum. On the other hand, beam-induced changes in electron microscope provide a simple means to understand the phase transition and reduction behaviour of the studied materials in a non-chemical ambient (high vacuum).

In this short paper, we report our first observation of electron beam induced changes in typical transition metal oxides
$\mathrm{V}_{2} \mathrm{O}_{5}, \mathrm{MoO}_{3}$ and $\mathrm{TiO}_{2}$ (anatase), studied by means of electron energy-loss spectroscopy (EELS) and of electron diffraction. In these three oxides, the metal atoms are in maximum valence. The energy-loss near edge structure (ELNES) of metal and oxygen atoms provide "fingerprints" of the changes in the oxidation state, in the chemical bonding and in the co-ordination of the detected species.

\section{Experimental}

Commercial powders of $\mathrm{V}_{2} \mathrm{O}_{5}, \mathrm{MoO}_{3}$ and $\mathrm{TiO}_{2}$ from Fluka $\mathrm{GmbH}$ were used. For TEM investigations, all oxides were crushed gently in carbon tetrachloride and dispersed onto a holey carbon film supported by a copper mesh grid. A Philips CM200 FEG electron microscope, operating at $200 \mathrm{kV}$ and equipped with GATAN imaging filters GIF100 for EELS measurement, was used. The high vacuum of the specimen chamber was kept lower than $10^{-7}$ Torr. All of the electron irradiation was performed at a current density of 3 $\mathrm{A} / \mathrm{cm}^{2}$. 


\section{Results and discussion}

1. $\mathrm{V}_{2} \mathrm{O}_{5}$

$\mathrm{V}_{2} \mathrm{O}_{5}$ crystallises in an orthorhombic structure with vanadium in the $\mathrm{V}^{5+}$ state. Vanadium $L$-edges and oxygen $K$ edges are used to monitor the electron irradiation induced electronic changes in $(001)$ orientated $\mathrm{V}_{2} \mathrm{O}_{5}$ crystals. A series of results after different irradiation times is shown in Fig. 1.

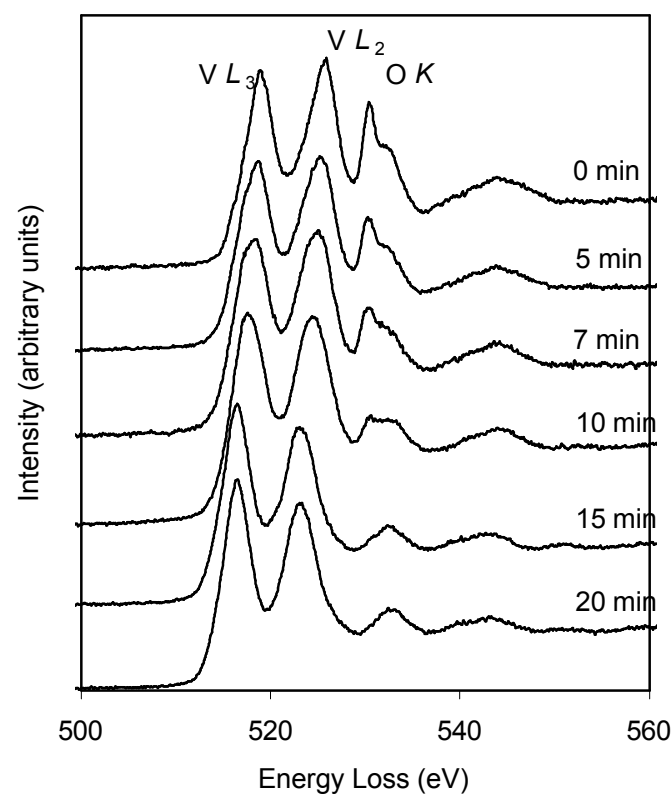

Fig. 1: $\mathrm{V} 2 p$ and $\mathrm{O} 1 s$ ELNES of $\mathrm{V}_{2} \mathrm{O}_{5}$ as a function of irradiation time. Spectra are offset for better distinction. The electron current density was $3 \mathrm{~A} / \mathrm{cm}^{2}$.

The initial spectrum, characterised by the vanadium $2 p \rightarrow$ $3 \mathrm{~d}$ transition (V $L$-edge) and the oxygen $1 s \rightarrow 2 p$ transitions (O $K$-edge), shows a decrease of the intensity of the oxygen $K$ signal with prolonged beam irradiation. Since this intensity is proportional to the number of oxygen atoms in the irradiated area, the decrease in intensity suggests a preferential loss of oxygen from the crystal lattice. The positions of the $\mathrm{V} L_{3}$ peak shifts from $519 \mathrm{eV}$ to lower energies, indicating a reduction of vanadium accompanied with the irradiation. After $20 \mathrm{~min}$, the $L_{3}$ peak reaches to $516.5 \mathrm{eV}$, which, according to a relationship between $L_{3}$-peak positions and oxidation states of vanadium in vanadium oxides [1], corresponds to $\mathrm{a} \mathrm{V}^{2+}$ state. We conclude that vanadium is reduced from $\mathrm{V}^{5+}$ to $\mathrm{V}^{2+}$.

The accompanying structural changes are revealed as changes in electron diffraction patterns. The patterns from initial $\mathrm{V}_{2} \mathrm{O}_{5}$ and from the final product are shown in Figs.2.
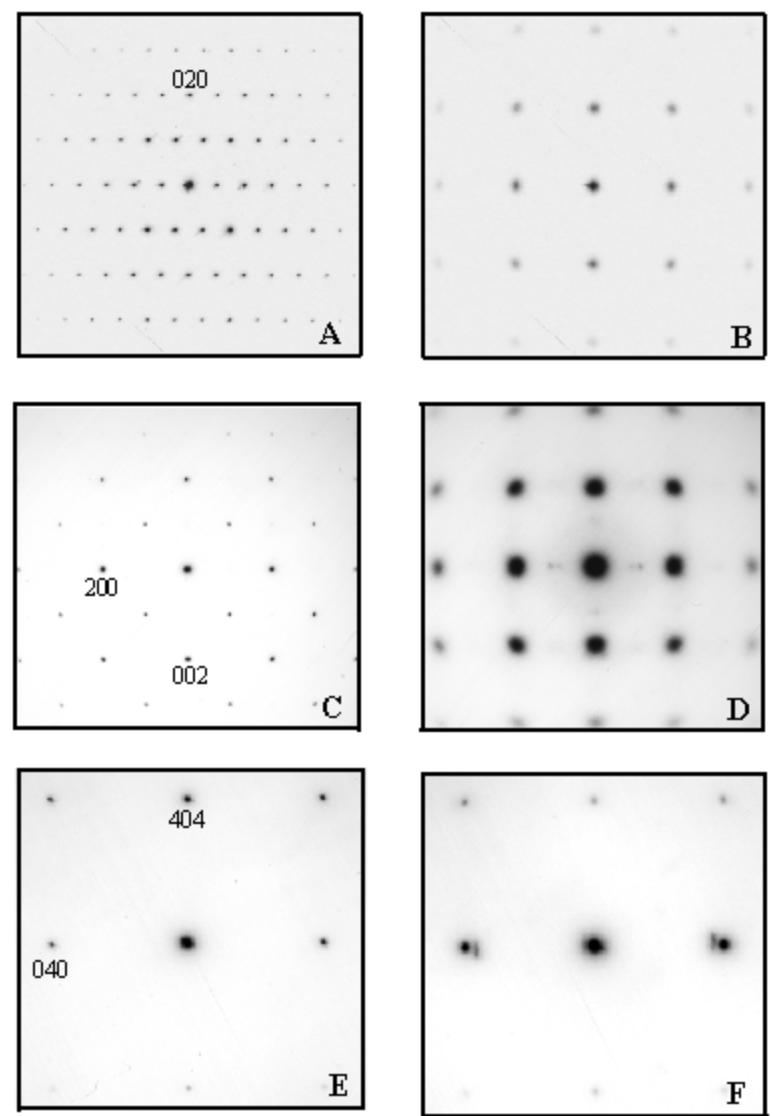

Fig. 2: Electron diffraction patterns. A: from 001-orientated $\mathrm{V}_{2} \mathrm{O}_{5}$; B: after 20 min irradiation; $\mathrm{C}$ : from 010-orientated $\mathrm{MoO}_{3}$; D: after 50 min irradiation; E: from 10-1-ortientated $\mathrm{TiO}_{2}$ (anatase); F: after 30 min irradiation.

The lattice parameters determined from the diffraction pattern after 20 min irradiation are $\mathrm{a}=\mathrm{b}=0.410 \mathrm{~nm}$ which are in good agreement with the lattice parameter of VO $(0.412$ $\mathrm{nm}$ ), supporting a structural change from orthorhombic $\mathrm{V}_{2} \mathrm{O}_{5}$ to cubic $\mathrm{VO}$ (vanadium in $\mathrm{V}^{2+}$ state).

\section{2. $\mathrm{MoO}_{3}$}

In the orthorhombic structure of $\mathrm{MoO}_{3}$, molybdenum is in the $\mathrm{Mo}^{6+}$ state. Initially the main features of energy-loss spectra are the Mo $M_{23}$ doublet peaks at about $400 \mathrm{eV}$ (due to the transition of Mo $3 p$ electrons to the unoccupied $4 d$ states) and the $\mathrm{O} K$ doublet peaks at about $520 \mathrm{eV}$ (due to transitions of $\mathrm{O} 1 s$ electron to the co-valence mixed states derived from the $\mathrm{O} 2 p$ and Mo $4 d$ states). With electron irradiation we observe, however, a strong decrease of the intensity of $\mathrm{O} K$-edge, as shown in Fig. 3, indicating also a preferential removal of oxygen atoms from crystal lattice. 


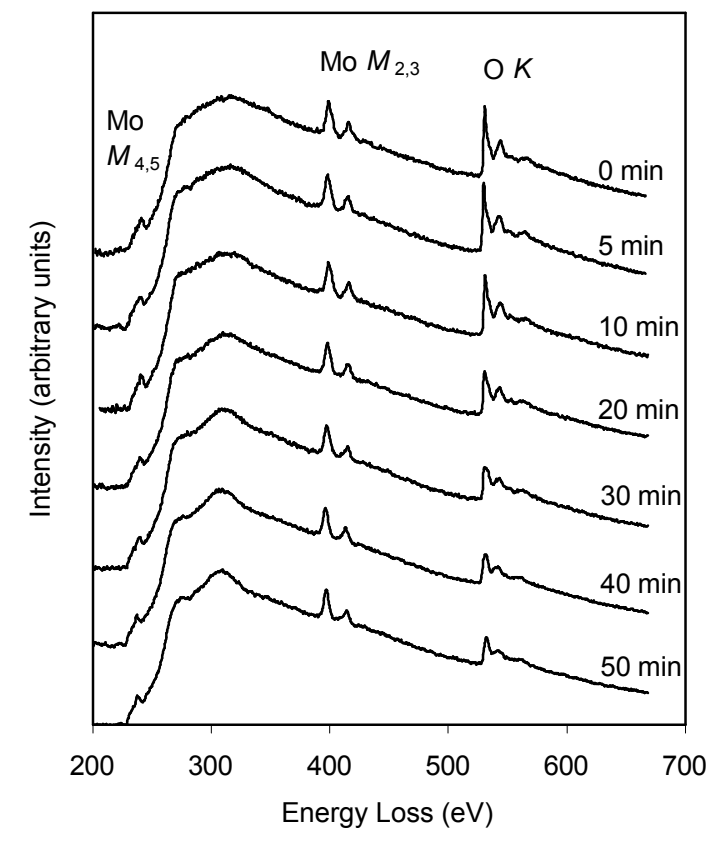

Fig. 3: Mo $3 d$ and $\mathrm{O} 1 s$ ELNES of $\mathrm{MoO}_{3}$ as a function of irradiation time. Spectra are offset for distinction. The electron current density was $3 \mathrm{~A} / \mathrm{cm}^{2}$.

The maximum of $M$-edges shifts slightly towards lower energies, but not as significant as the corresponding chemical shifts of $\mathrm{V} L$-edges. Comparison of the spectrum reduced by 60 min irradiation with that of pure $\mathrm{MoO}_{2}$ and Mo indicates that the final states is lower than that of $\mathrm{Mo}^{4+}$. In insitu characterisation of catalytic reaction of $\mathrm{MoO}_{3}$ a final reduced phase of $\mathrm{MoO}_{2}\left(\mathrm{Mo}\right.$ in $\mathrm{Mo}^{4+}$ state) was detected [2]. It was reported that under the electron beam $\mathrm{MoO}_{3}$ could be reduced to metallic molybdenum [3]. Our observation, however, cannot confirm this finding since after $60 \mathrm{~min}$ of irradiation we can still detect oxygen signal, although the diffraction pattern changes to the one as from f.c.c. metal (Figs. 2). The lattice parameters, calculated from the diffraction patterns, are $\mathrm{a}=\mathrm{c}=0.408 \mathrm{~nm}$. Among the known molybdenum oxides, no phases with such lattice parameters can be identified.

\section{3. $\mathrm{TiO}_{2}$ (anatase)}

The ELNES characteristic of $\mathrm{Ti}$ in $\mathrm{TiO}_{2}$ (anatase) are the two doublet peaks that stem from the electron transition from the Ti $2 p_{3 / 2}$ and $2 p_{1 / 2}$ to the Ti $3 d$ orbitals which in turn split into $t_{2 g}$ and $e_{g}$ orbitals due to the octahedral symmetry.
Ti-L edge of $\mathrm{TiO}_{2}$

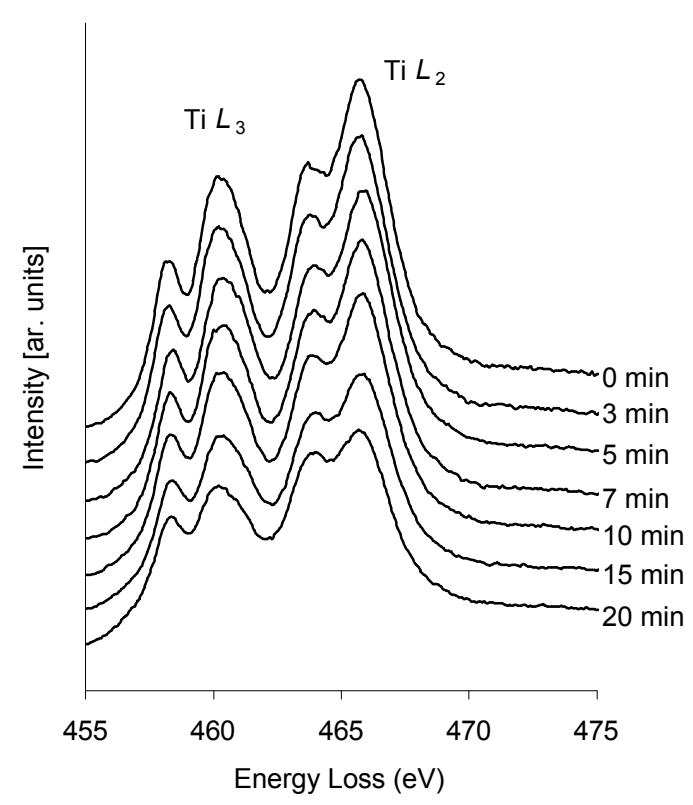

Fig. 4: Ti $2 p$ ELNES of $\mathrm{TiO}_{2}$ (anatase) as a function of irradiation time. Spectra are offset for distinction. The electron current density was $3 \mathrm{~A} / \mathrm{cm}^{2}$.

As shown in Fig.4, the shape of $L_{2,3}$ edges changes slightly due to the electron irradiation, but no chemical shifts can be detected. Furthermore, no noticeable changes in the intensity of O $K$-edge can be observed (Fig. 5).

O K-edge of $\mathrm{TiO}_{2}$

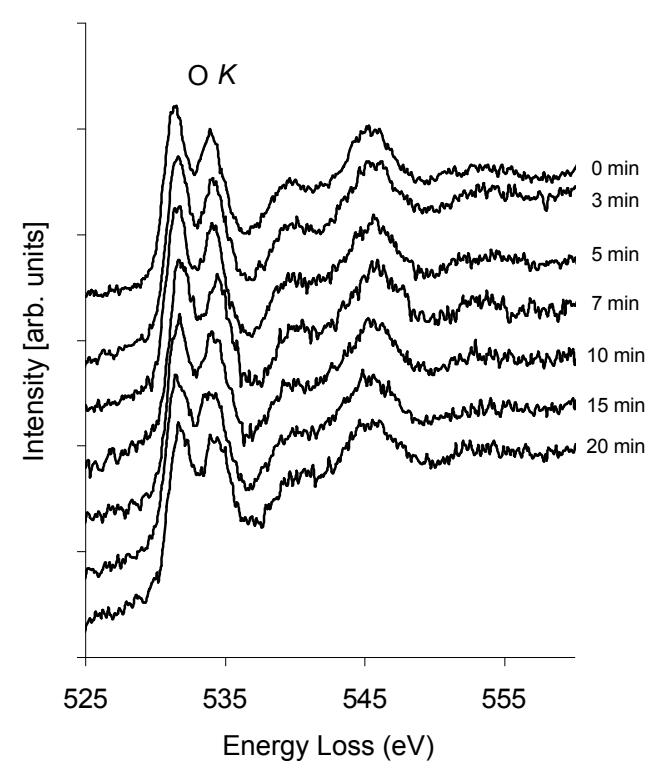

Fig. 5: $\mathrm{O} 1 s$ ELNES of $\mathrm{TiO}_{2}$ (anatase) at various irradiation times. Spectra are offset for distinction. The electron current density was $3 \mathrm{~A} / \mathrm{cm}^{2}$. 
The electron diffraction patterns from initial anatase and after 30 min irradiation are shown in Figs.2. In contrast to other two oxides, the main symmetry prevails during the electron irradiation, with weak additional spots appear.

\section{Conclusion}

Our experiments reveal quite different behaviour of maximal valence transition metal oxides under electron beam irradiation. While $\mathrm{TiO}_{2}$ (anatase) is quite electron beam-resistant, $\mathrm{V}_{2} \mathrm{O}_{5}$ and $\mathrm{MoO}_{3}$ show dramatic change under electron beam. Orthorhombic $\mathrm{V}_{2} \mathrm{O}_{5}$ can be changed to cubic VO. $\mathrm{MoO}_{3}$ can be reduced to a phase with oxidation state less than that in $\mathrm{MoO}_{2}$. The results show how critical the electron bombardment is when transition metal oxide is studied in highvoltage electron microscope.

\section{Acknowledgement}

The work is supported by SFB 546 of the Deutsche Forschungsgemeinschaft (DFG).

\section{References}

[1] Chen JG, Kim CM, Frühberger B, De Vries BD, and Touvelle MS (1994) Surf Sci 321: $145-155$

[2] Ressler T, Jentoft RE, Wienold J, Günter MM., Timpe O (2000) J Phys Chem B 104: 6360-6370

[3] Buckett MI, Strane J, Luzzi DE, Zhang JP, Wessels BW, and Marks LD (1989) Ultramicroscopy 29: 217-227 